\section{Histamine concentration is involved in canine valvular disease}

\author{
Mitsuhiro Isaka, Masahiko Befu, Nami \\ Matsubara, Mayuko Ishikawa, Yurie \\ Arase, Shinichi Namba \\ Marble Veterinary Medical Center, \\ Fujisawa, Japan
}

\begin{abstract}
It has been known since many years that there are histamine receptors $(\mathrm{H})$ in the heart. Histamines display chronotropic and inotropic activity, cardiovascular diseases, and are thought to be a systemic inflammatory disease. During heart failure, the histamine concentration is elevated. In addition, $\mathrm{H} 2$ blockers prolonged the survival period for human patients with heart failure. The aim of this study was to evaluate whether blood concentration of histamine is associated with canine valvular disease (CVD). The histamine concentrations of dogs with CVD are significantly higher than those of healthy dogs. The histamine concentration gradually increases during CVD and is highly correlated with the grade of heart murmur. In conclusion, the histamine concentration was higher in the population of dogs with CVD compared with the healthy controls. Although the etiopathogenesis of CVD is complex and incompletely understood, it likely involves histamine. Ultimately additional studies are required to determine whether histamine blockers might be useful for the management of dogs with cardiac valvular disease.
\end{abstract}

\section{Introduction}

It has been known for many years that there are histamine receptors $(\mathrm{H})$ in the heart. ${ }^{1}$ Histamine receptors are divided into four subtypes $\mathrm{H} 1, \mathrm{Hs}, \mathrm{H} 3$ and $\mathrm{H} 4 .{ }^{2}$ Histamine and its antagonists, such as cimetidine, have direct chronotropic and dromotropic effects. ${ }^{3}$ Histamine also modulates norepinephrine release from the cardiac sympathetic nerve, such as endothelin. ${ }^{4-6}$ Thus, in essence, histamine is a true neuro-hormone.

Mast cells are found in the heart and are known to release histamine. ${ }^{7}$ Mast cells and histamine release have been implicated in human heart disease.7,8 In addition to histamine release, mast cells appear to be a unique source of renin. ${ }^{9}$ Thus, in some cases, histamine might contribute to the local activation of the renin-angiotensin-aldosterone system in the heart and all of the potential adverse consequences of that particular neuro-hormonal system. Using an isolated Langendorff preparation, Mackins et al. ${ }^{10}$ demonstrated that cardiac mast cell-derived renin promotes local angiotensin formation, norepinephrine release, and arrhythmias in an ischemic/reperfusion model and that mast cell stabilization with cromolyn or iodoxamide attenuates this process. The elevation of serum histamine and mast cells accumulation in the heart were observed during heart failure in human patients. ${ }^{7}$ In addition, $\mathrm{H} 2$ blockers have been demonstrated to prolong survival time during heart failure in human patients. ${ }^{11}$ Collectively, these data suggest that histamine might play an important role in heart disease. However, to the best of our knowledge, there are no reported studies that have investigated the role of histamine in canine valvular disease (CVD). Therefore, the aim of this study was to evaluate the histamine serum concentrations of affected and unaffected dogs.

\section{Materials and Methods}

Client-owned dogs suffering from chronic valvular disease (mitral regurgitation; $n=21$ ) and healthy controls $(n=7)$ were examined. Summary of clinical data of the experimental groups are in Table 1. Disease diagnoses were established by physical examination, electrocardiography, thoracic radiography, 2-D and Doppler echocardiography, as well as blood examination (complete blood counts, biochemistry, C-reactive protein). All of the animals were negative for Dirofilaria immitis infection. All patients did not have concurrent diseases at the time of measurement for plasma histamine. The dogs suffering from valvular disease were administered enalapril or ramipril, spironolactone, furosemide or torasemide, pimobendan, or isosorbide dinitrate, dependent on the condition of the disease. Serum histamine concentrations were measured in all animals. Control: Cavalier King Charles Spaniel $n=3$, T. Poodle $n=1$, Mix breed $n=1$, G. Retriever $n=1$, Pomeranian $n=1$. For the CVHD grades, class I: Cavalier King Charles Spaniel $n=2$, Pug $n=1$, Papillon $n=1$, Mix breed $n=1$, Pomeranian $n=1$. Class II: Norfolk Terrier $n=1$, Chihuahua $n=1$, Maltese $\mathrm{n}=1$, Cavalier King Charles Spaniel $\mathrm{n}=1$, Shih Tzu $n=1$, Mix breed $n=1$. Class III: Chihuahua $\mathrm{n}=2$, Shih Tzu $\mathrm{n}=2$, French Bulldogs $\mathrm{n}=1$, Cavalier King Charles Spaniel $n=2$, Beagle $\mathrm{n}=1$, Mix breed $\mathrm{n}=1$. All patients did not have concurrent diseases at the time of measurement for plasma histamine. The dogs suffering from valvular disease were administered enalapril or ramipril, spironolactone, furosemide or torasemide, pimobendan, or
Correspondence: Mitsuhiro Isaka, Marble Veterinary Medical Center, 4-1-6 Ishikawa Fujisawa, Kanagawa 252-0815, Japan.

Tel. +81.466 .862 .080 - Fax: +81.466 .860 .558 .

E-mail: isakam7@gmail.com; mitsuhiro_isaka @mcvj.com

Key words: histamine, dogs, valvular disease.

Contributions: the authors contributed eqally.

Received for publication: 12 October 2013.

Accepted for publication: 26 November 2013.

This work is licensed under a Creative Commons Attribution NonCommercial 3.0 License (CC BYNC 3.0).

(C) Copyright M. Isaka et al., 2014

Licensee PAGEPress srl, Italy

Veterinary Science Development 2014; 4:5123

doi:10.4081/vsd.2014.5123

isosorbide dinitrate, dependent on the condition of the disease. The serum samples were maintained at $-20^{\circ} \mathrm{C}$ until the experiments were performed. A commercial enzyme-linked immunosorbent assay (ELISA) kit (SPI Bio, Bertin Pharma Inc., Montigny le Bretonneux, France) was utilized, and the histamine concentrations of the healthy and CVD samples were compared and also investigated using the correlation between the ISACHC (International Small Animal Cardiac Heart Council) classification and heart murmur intensity. 12 The data are expressed as the mean \pm standard error. An unpaired t- test was used for comparisons between the healthy and valvular heart disease dogs (Figure 1A). An ANOVA post hoc Bonferroni test was used to compare the healthy and ISACHC-classified dogs (Figure 1B). We also evaluated the correlation between the grade of heart murmur and the histamine concentration (Figure 2). A value of $\mathrm{P}<0.05$ was considered statistically significant. All analyses were performed using commercial statistical software (StatMate v. 4.01).

Twenty-eight client-owned dogs were enrolled in this study, which included 7 healthy control dogs and 21 dogs suffering from CVD. The CVD dogs were divided into ISACHC classes I, II and III. The control group dogs $(n=7)$ had body weights of $9.2 \pm 2.5 \mathrm{~kg}$ and were $116.3 \pm 12.6$ months old in age. Class I $(n=6)$ had body weights of $7.3 \pm 1.2 \mathrm{~kg}$ and were $105.5 \pm 26.9$ months old in age, Class II $(n=6)$ had body weights of $6.3 \pm 1.0 \mathrm{~kg}$ and were $102.2 \pm 18.3$ months old in age, and Class III $(\mathrm{n}=9)$ had body weights of $8.0 \pm 2.7 \mathrm{~kg}$ and were $129.0 \pm 7.7$ months old in age.

The dogs suffering from CVD had higher histamine concentrations $(63.2 \pm 25.2 \mathrm{nM})$ 
compared to the healthy control dogs $(13.1 \pm 7.6$ $\mathrm{nM}, \mathrm{P}<0.01$; Figure 1A). Among the ISACHC grades, Class 1: $\mathrm{n}=6$ : $45.4 \pm 16.1(\mathrm{nM})$, Class 2: $\mathrm{n}=6: 53.8 \pm 27.5(\mathrm{nM})$, and Class $3: \mathrm{n}=9$ : $80.4 \pm 20.3(\mathrm{nM})$; Figure 1B. Thus, the histamine concentration gradually elevated dependent on the ISACHC grade. There was significant relationship between murmur grade and histamine concentration in the dogs suffering from CVD $(r=0.559 ; \mathrm{P}<0.01$; Figure 2). These results indicate that the histamine concentration was higher in the CVD population of dogs compared to the healthy controls. Although it appears clear that a genetic basis exists for CVD development, the results of this study support the growing body of evidence that inflammatory mediators may contribute to the deterioration of heart valves over time. ${ }^{13}$

\section{Discussion}

In a canine heart failure model, histamine expression was elevated, and mast cells were collected in cardiomyocytes.$^{8}$ In addition, the blood histamine concentration is elevated during human heart failure. ${ }^{7}$ A number of studies have also investigated whether inflammation plays a role in the progression of CVD and cardiac ischemia/reperfusion.4,13-16 Collectively, the phenomena involved in this study might be related. The results of this study suggest that there may be an underlying systemic inflammatory process involved in heart disease. However, in our study, we did not evaluate the associations among histamine and other inflammatory substances. Thus, an elevated histamine concentration might be associated with other substances. In our study, there was a good correlation between heart murmur intensity and histamine concentration, and this phenomenon is not similar to the results found between heart murmur and C-reactive protein in dogs. 13 This discrepancy is not clear; however, a proinflammatory reaction might be associated with heart disease in dogs.

In the clinical setting, a combination of beta blockers and blocking histamine production (H2 blocker) showed a significant improvement in cardiac function, as demonstrated by echocardiography, compared to the administration of beta blocker alone. 8 Thus, histamine blockers might be useful for the treatment of canine valvular disease in the future.

\section{Conclusions}

In conclusion, the histamine concentration was higher in the population of dogs suffering from CVD compared to the healthy controls. The pathogenesis of CVD is complex, not fully
Table 1. Clinical characteristics of each experimental group.

\begin{tabular}{lcccc} 
& $\begin{array}{c}\text { Control } \\
(\mathrm{n}=7)\end{array}$ & $\begin{array}{c}\text { ISACHC I } \\
(\mathrm{n}=6)\end{array}$ & $\begin{array}{c}\text { ISACHC II } \\
(\mathrm{n}=6)\end{array}$ & $\begin{array}{c}\text { ISACHC III } \\
(\mathrm{n}=9)\end{array}$ \\
Body weight (kg) & $9.2 \pm 2.5$ & $7.3 \pm 1.2$ & $6.3 \pm 1.0$ & $8.0 \pm 2.7$ \\
Age (months) & $116.3 \pm 12.6$ & $105.5 \pm 26.9$ & $102.2 \pm 18.3$ & $129.0 \pm 7.7$ \\
\hline Female/Male (n) & $4 / 3$ & $0 / 6$ & $2 / 4$ & $7 / 2$ \\
Heart rate (bpm) & $114.8 \pm 7.9$ & $130.7 \pm 14.3$ & $101.3 \pm 6.0$ & $134.3 \pm 8.5$ \\
\hline VHS (v) & $10.3 \pm 0.3$ & $10.1 \pm 0.2$ & $10.6 \pm 0.3$ & $11.2 \pm 0.4$ \\
La/Ao & $1.57 \pm 0.1$ & $1.71 \pm 0.1$ & $1.92 \pm 0.16$ & $2.24 \pm 0.15$ \\
\hline
\end{tabular}

ISACHC, International Small Animal Cardiac Heart Council; VHS, vertebral heart size; La/Ao, ratio of left atrium and root of aorta.

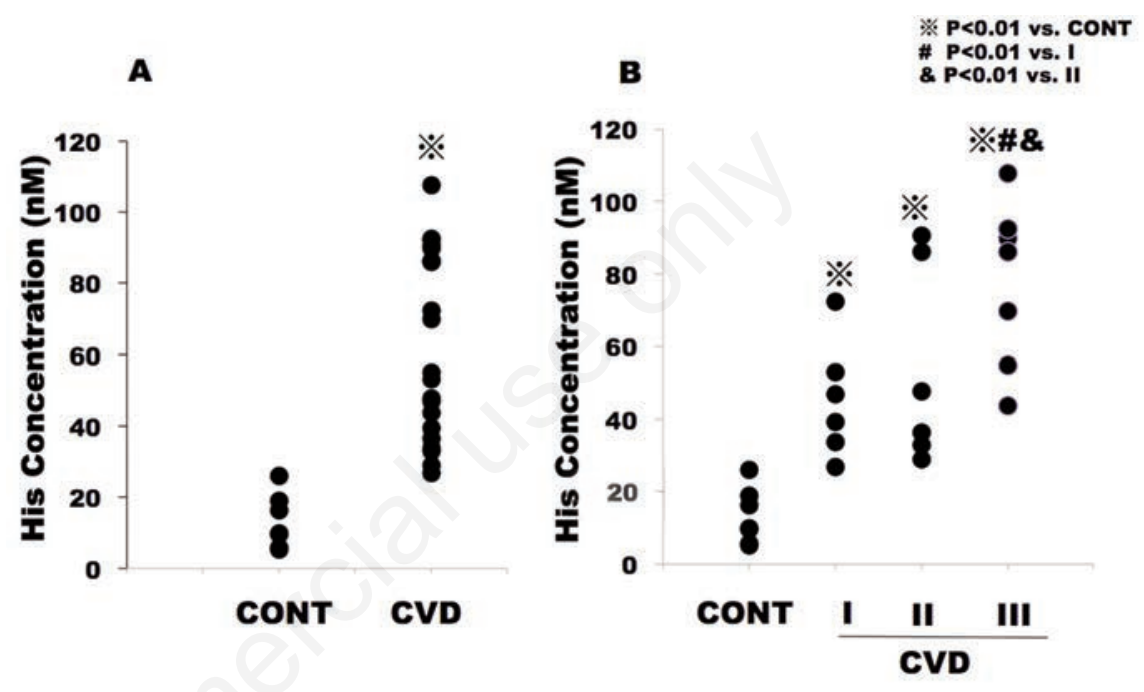

Figure 1. A) Serum Concentrations of Histamine (nM) in Healthy (CONT) and Chronic Valvular Disease Dogs (CVD) $(\mathbf{P}<\mathbf{0 . 0 1})$. B) The Association between Serum Histamine Concentration and grades based on the International Small Animal Cardiac Heart Council (ISACHC) Classification.

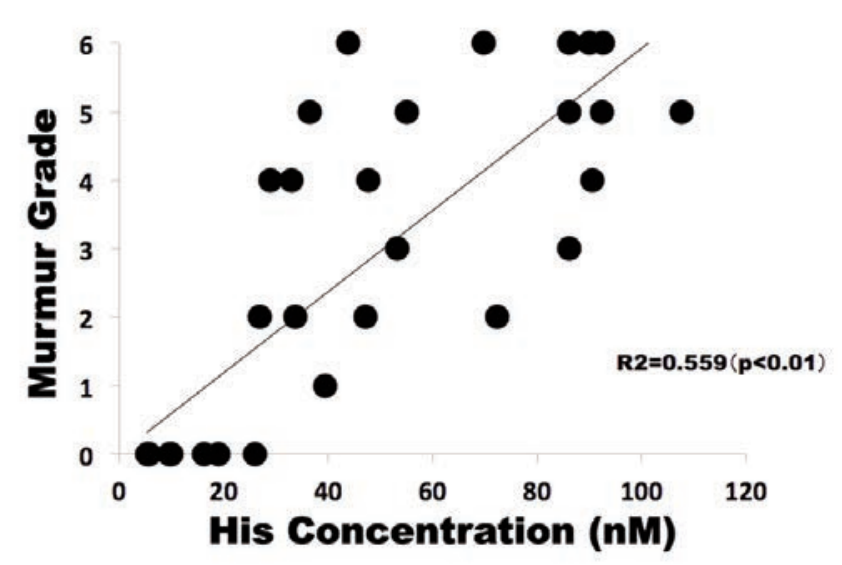

Figure 2. The Correlation between Histamine Concentration and Heart Murmurs. There is a significant correlation between intensity of heart murmur and serum histamine concentration. 
understood, and likely relates to certain systemic inflammations, such as histamine production. Ultimately more studies will be required to determine whether histamine measurements can be useful in assessing or managing dogs suffering from cardiac valvular disease.

\section{References}

1. Levi R, Kuye J0. Pharmacological characterization of cardiac histamine receptors: sensitivity to H1-receptor antagonists. Eur J Pharmacol 1974;27:330-8.

2. Saligrama N, Noubade R, Case LK, et al. Combinatorial roles for histamine $\mathrm{H} 1-\mathrm{H} 2$ and $\mathrm{H} 3-\mathrm{H} 4$ receptors in autoimmune inflammatory disease of the central nervous system. Eur J Immunol 2012;42:153646.

3. Hageman GR, Urthaler F, Isobe JH, James TN. Chronotropic and dromotropic effects of histamine on the canine heart. Chest 1979:597-604.

4. Isaka M, Kudo A, Imamura M, et al. Endothelin receptors, localized in sympathetic nerve terminals of the heart, modulate norepinephrine release and reperfusion arrhythmias. Basic Res Cardiol
2007:102;154-62.

5. Isaka M, Imamura M, Sakuma I, et al. Cardiopulmonary bypass influences the plasma levels of calcitonin gene-related peptides in dogs: effects of hemofiltration and hemodilution. Res Vet Sci 2007;82: 110-4.

6. Imamura M, Poli E, Omoniyi AT, Levi R. Unmasking of activated histamine H3receptors in myocardial ischemia: their role as regulators of exocytotic norepinephrine release. J Pharmacol Exp Ther 1994;271:1259-66.

7. Francis GS, Tang WH. Histamine, mast cells, and heart failure: is there a connection? J Am Coll Cardiol 2006;48:1385-6.

8. Takahama H, Asanuma H, Sanada S, et al. A histamine $\mathrm{H}_{2}$ receptor blocker ameliorates development of heart failure in dogs independently of $\beta$-adrenergic receptor blockade. Basic Res Cardiol 2010;105:78794.

9. Silver RB, Reid AC, Mackins CJ, et al. Mast cells: a unique source of renin. Proc Natl Acad Sci USA 2004;101:13607-12.

10. Mackins CJ, Kano S, Seyedi N, et al. Cardiac mast cell-derived renin promotes local angiotensin formation, norepinephrine release, and arrhythmias in ischemia/reperfusion. $\mathrm{J}$ Clin Invest 2006;116:1063-70.
11. Kim J, Ogai A, Nakatani S, et al. Impact of blockade of histamine $\mathrm{H} 2$ receptors on chronic heart failure revealed by retrospective and prospective randomized studies. J Am Coll Cardiol 2006;48:1378-84.

12. Fox PR, Sisson D, Moise NS. Recommendations for diagnosis of heart disease treatment of heart failure in small animals. In: ISACHC. Textbook of canine and feline cardiology, principles and clinical practice, 2nd ed., Philadelphia: W.B. Saunders; 1999. pp. 883-901.

13. Rush JE, Lee ND, Freeman LM, Brewer B. C-reactive protein concentration in dogs with chronic valvular disease. J Vet Intern Med 2006;20:635-9.

14. Prosek R, Sisson DD, Oyama MA, et al. Plasma endothelin-1 immunoreactivity in normal dogs and dogs with acquired heart disease. J Vet Intern Med 2004;18:840-4.

15. Pedersen HD, Falk T, Häggström J, et al. Circulating concentrations of insulin-like growth factor-1 in dogs with naturally occurring mitral regurgitation. J Vet Intern Med 2005;19:528-32.

16. Zois NE, Moesgaard SG, Kjelgaard-Hansen $\mathrm{M}$, et al. Circulating cytokine concentrations in dogs with different degrees of myxomatous mitral valve disease. Vet $\mathrm{J}$ 2012;192:106-11. 